\title{
Diagnosis and Treatment of Insufficient Dialysis Vascular Access
}

\section{Leitliniengerechte Diagnostik und Therapie bei insuffizienten Dialyseshunts}

Authors

Affiliation
L. Kamper, T. D. Faizy, P. Haage

Diagnostic and interventional Radiology, HELIOS Clinic Wuppertal, University Hospital Witten/Herdecke, Wuppertal, Germany

\section{Key words \\ - vascular \\ - angioplasty \\ - ultrasound \\ - venography \\ - CT angiography}

received 27.6.2014 accepted $\quad 3.12 .2014$

Bibliography

DOI http://dx.doi.org/

$10.1055 / \mathrm{s}-0034-1398826$

Published online: 2.2 .2015

Fortschr Röntgenstr 2015; 187:

360-371 @ Georg Thieme

Verlag KG Stuttgart · New York . ISSN 1438-9029

\section{Correspondence \\ Dr. Lars Kamper}

Diagnostic and Interventional Radiology, HELIOS-Klinikum

Wuppertal, University Witten/ Herdecke

Heusner Str. 40

42283 Wuppertal

Germany

Tel.: ++ 49/2 02/8962565

Fax: $++49 / 202 / 8962565$

lars.kamper@helios-kliniken.de

\section{Abstract \\ $\nabla$}

A wide variety of diagnostic and minimally invasive modalities is currently available to radiologists for providing sufficient medical care for dialysis patients. In addition to clinical examination, ultrasound is the first line imaging method in suspected dialysis vascular access dysfunction. The percutaneous approach is the primarily recommended therapeutic option in many cases. This article reviews the current diagnostic and therapeutic methods in dialysis vascular access insufficiency.

Key Points:

- Vascular access dysfunction is often elicited by stenosed vessels, which are regularly associated with concomitant thrombosis.

- Stenoses are commonly observed in the anastomosis, vascular access vein and also in the central venous system.

- Beside the clinical examination, sonography is the diagnostic method of first choice.

- Percutaneous balloon dilatation frequently is the first treatment option.

Citation Format:

- Kamper L, Faizy TD, Haage P. Diagnosis and Treatment of Insufficient Dialysis Vascular Access. Fortschr Röntgenstr 2015; 187: 360-371

\section{Zusammenfassung}

\section{$\nabla$}

Zur adäquaten medizinischen Betreuung des Dialysepatienten stehen dem Radiologen heute eine Vielzahl von diagnostischen und minimalinvasiven Methoden zur Verfügung. Neben der klinischen Untersuchung ist die Sonografie die bildgebende Methode der ersten Wahl bei vermuteter Dialyseshuntdysfunktion. Therapeutisch stellt die perkutane Behandlung oftmals die primär emp- fohlene Option dar. Die vorliegende Arbeit gibt einen Überblick über den derzeitigen Stand diagnostischer und therapeutischer Verfahren bei Malfunktion des Dialyseshunts.

\section{Introduction \\ $\checkmark$}

Proper vascular access function is required for efficient dialysis. With an unchanged incidence of patients undergoing renal replacement therapy and an increasing life expectancy, an increase in the insufficiency of dialysis accesses has accordingly been seen [1]. By maintaining long-term function, the use of dialysis catheters associated with a higher mortality rate can be avoided [2].

The most common complications in dysfunctional AV fistulas are stenosis of the vascular access vessels (14-42\%), thrombosis (17$25 \%)$, and steal syndrome (2-8\%) [3]. In the case of AV fistulas, stenoses often occur in the region of the anastomosis [4] and in the course of the vascular access vein [5]. Moreover, dialysis patients develop central venous stenoses often as a reaction to wall trauma associated with the dialysis catheter and as a result of the arterialized flow conditions in the venous circulation [6] vascular access thromboses typically arise as a result of these stenoses and can occur as a segmental thrombosis or extensive thrombotic occlusion [3]. Vascular access insufficiency is ideally diagnosed and treated in the framework of continuous interdisciplinary cooperation between nephrologists, vascular surgeons, and interventional radiologists [7-9].

Duplex sonography is the method of choice for imaging insufficient dialysis vascular access. Central vessels that cannot always be viewed sonographically can be visualized via angiography. In addition, diagnosis and treat- 
ment can be performed in one session. Sectional imaging techniques are used as secondary methods. Computed tomography (CT) is suitable for visualizing central veins and the complete vascular tree in the case of an inconsistent sonographic finding. Gadolinium-enhanced MRI is not recommended for visualizing vascular access vessels due to the potential risk of nephrogenic systemic fibrosis (NSF) in dialysis patients.

In the case of an insufficient dialysis vascular access, prompt treatment is decisive for being able to avoid a possible loss of the vascular access and the use of a temporary dialysis catheter. A number of angiographic techniques are available for this purpose.

Elastic or rigid stenoses can be treated by conventional PTA and stent implantation as well as by cutting balloon, for example [10, 11]. To prevent recurrent stenoses, the use of drug-coated balloons is increasingly being reported [12, 13]. There are currently no randomized studies or even larger case series showing a superior long-term patency rate for endovascular brachytherapy $[14,15]$ and cryoplasty [16].

In addition to thrombolysis via medication, thrombosed AV fistulas and vascular access grafts can be treated in a combined pharmacomechanical manner or purely mechanically with thrombectomy catheters [17] or hydrodynamic systems [18].

The present article is based on the currently available published guidelines including the European Best Practice Guidelines (EBPG) and KDOQI Guidelines of the National Kidney Foundation [19-23], the upcoming guidelines of the European Society for Vascular Surgery and a search of the literature in the database info system (DBIS) Science Citation Index Expanded (SCI Expanded/ Web of Science), and in the PubMed literature database.

\section{Diagnosis \\ $\nabla$}

\section{Ultrasound}

Due to its wide availability and lack of invasiveness, duplex sonography is the most widely used vessel visualization method. As a cost-effective method, it allows evaluation of vascular access maturity and diagnosis of complications in experienced hands [24-27]. As a result of sonographic stenosis detection, flow measurement and the diagnosis of thrombotic occlusions, the cause of a vascular access insufficiency can usually be reliably identified on ultrasound [28, 29]. In addition to stenosis detection, sonographic followup after treatment is readily possible ( $\bullet$ Fig. 1 ). Therefore, ultrasound has become established as the primary imaging method in the case of suspicion of vascular access insufficiency $[8,21,26]$.

Malik et al. reported that long-term follow-up via threemonth duplex sonography can significantly extend the patency rate of dialysis vascular access [30]. The authors state that this was achieved by earlier detection of stenosis with correspondingly faster treatment.

Amin et al. stated that a flow rate of less than $600 \mathrm{ml} / \mathrm{min}$ within implanted PTE grafts is a predictor of significant stenoses [31]. However, there is no relationship between the blood flow within the vascular access graft and the length, location, and number of possible definable stenoses.
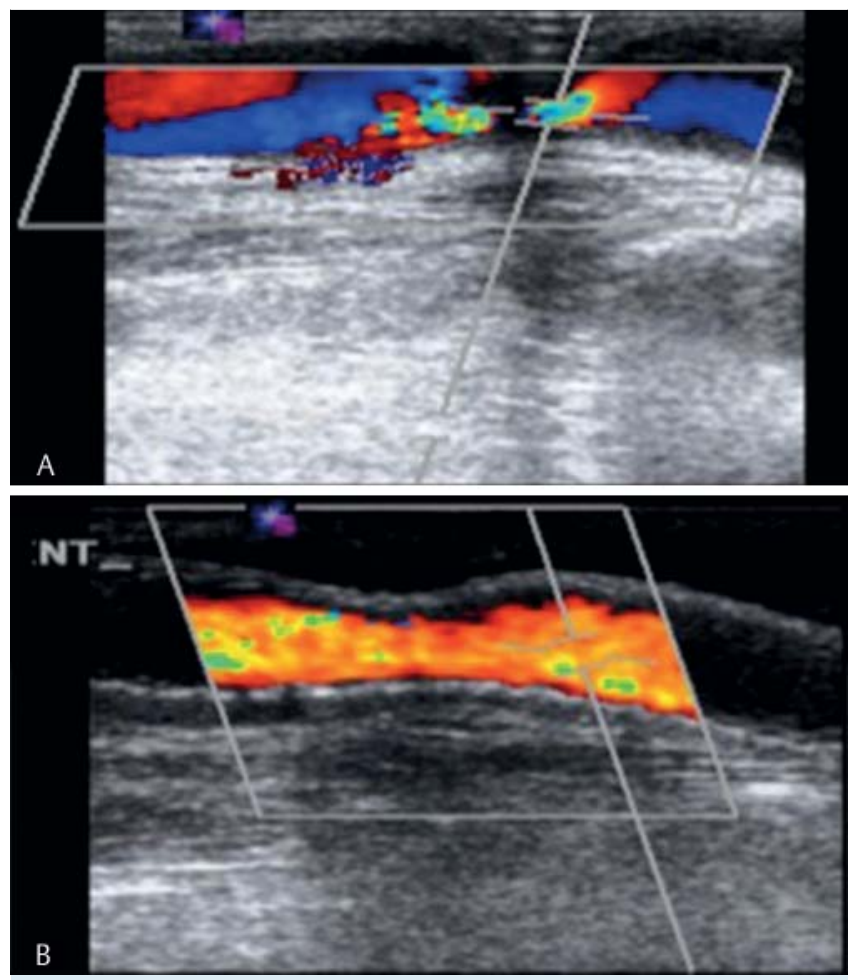

Fig. 1 Sonographic visualization of a venous stenosis with significantly accelerated blood flow (A) control after percutaneous therapy (B).

Although infections of vascular accesses and vascular access systems are typically diagnosed clinically, the extent of paravascular infections and possible consecutive thromboses can be diagnosed via ultrasound [32].

A disadvantage of the method is the dependence of the diagnostic quality on the experience of the examiner [33, 34]. Moreover, ultrasound examinations cannot be used to create a direct anatomical representation of the entire vascular tree [35].

However, experienced examiners can use the sonographic findings to create drawings for exact documentation of the vascular access, thus allowing intervention and operation planning [36].

However, evaluation of the central veins is still only possible on a limited basis [8]. Methods such as digital subtraction angiography are used for this purpose. This method allows better visualization of the central veins and the exclusion of central obstructions [21].

\section{Angiography}

Contrast angiography is the gold standard for the visualization of stenoses, occlusions, and aneurysms of dialysis vascular access and offers the advantage of complete vessel visualization. Digital subtraction angiography (DSA) requires radiation and contrast agent containing iodine or $\mathrm{CO}_{2}$. Purely diagnostic DSA without a therapeutic measure is not recommended for reasons of radiation hygiene [8]. DSA is used for preoperative mapping of vascular systems only in select cases. In particular, if central venous stenosis is suspected, DSA is clearly superior to ultrasound [8]. A further advantage is the possibility of parallel detection and treatment of stenoses. 
DSA allows periinterventional as well as postoperative evaluation of stenoses of the afferent and efferent legs of a dialysis vascular access and the detection of central thrombi. Any remaining stenoses [37] and central vein stenoses can also be detected in this manner [6]. Complete angiographic visualization, which can also be conducted in an ambulant setting [38], is performed via retrograde arterial fine-needle puncture of the brachial artery near the elbow or antegrade puncture of the efferent vascular access vein.

In the latter case of venous puncture, arteriovenous anastomosis via compression of the vascular access vein (preferably via blood pressure cuff) can be visualized via the retrograde contrast medium flow. Alternatively to arterial puncture, stenoses of the arterial branch in a more proximal position can be visualized via retrograde introduction of the catheter through the anastomosis into the native portion of the arterial vessel. Duijm and colleagues were able to achieve successful visualization with this technique in 21 of 22 patients with known arterial stenoses. Retrograde visualization was not possible in one patient since the anastomosis was impassable due to additional subtotal stenoses. Of the remaining patients, 18 could be successfully endovascularly treated [38].

The use of contrast agents containing iodine can result in a further limitation of kidney function in patients with terminal renal insufficiency. However, DSA can be performed by an experienced examiner with a very low contrast agent volume. Diluted iodine-containing contrast agent (e.g. 1:2 or $1: 3$ diluted in a physiological $\mathrm{NaCl}$ solution) with a contrast agent volume of $10 \mathrm{ml}$ to a maximum of $20 \mathrm{ml}$ is usually sufficient for diagnostic visualization.

Asif et al. showed that after application of $10-20 \mathrm{ml}$ of a low-osmolar contrast agent, 1 of 25 patients with severely limited renal function was able to undergo dialysis after 4 weeks [39].

To date, there is no conclusive evidence of the influence of contrast agent osmolarity on the development of contrastinduced nephropathy [40]. No significantly reduced nephrotoxicity was seen in a comparison of low-osmolar and iso-osmolar contrast agents in 324 patients with renal insufficiency undergoing percutaneous coronary intervention [41].
CO2 angiography represents an alternative to conventional DSA. Compared to DSA, CO2 angiography had a sensitivity of $97 \%$ and a specificity of $85 \%$ regarding the detection rate of central venous pathologies [42]. In addition, this method was successfully used for the diagnosis and intervention of dysfunctional dialysis vascular access [43]. In the selection of an arterial access, the potential risk of cerebral $\mathrm{CO} 2$ embolization must be taken into consideration. In principle, gadolinium-enhanced DSA is a further effective method for angiographic vessel visualization. However, this is not recommended due to the risk of nephrogenic systemic fibrosis in dialysis patients [44].

For treating dialysis vascular access, alternatives such as CT and MRI-guided interventions are currently neither more user-friendly nor more technically successful than angiography.

\section{Computed tomography}

CT angiography (CTA) should only be used to clarify vascular access insufficiency if a comparable method cannot be performed or other methods yielded inconclusive findings. In the case of suspicion of external vascular compression, paravascular soft tissues can be visualized, thus making it possible to diagnose malignant stenoses, for example. Examination should be performed in two consecutive contrast-enhanced series in the arterial and venous phase. Approximately $40-60 \mathrm{ml}$ of contrast agent are sufficient for modern CT protocols [45].

In particular, central venous thromboses can be effectively detected with CT ( $\bullet$ Fig. 2 ). CTA can provide important information for the further course of treatment also for recommended operative and interventional procedures. Some authors therefore refer to CTA as superior to purely diagnostic DSA for economic reasons [46]. CTA allows diagnosis of dysfunctional vascular access $[47,48]$ as well as simultaneous visualization of the entire vascular tree [49, 50]. Moreover, CTA is capable of diagnosing hemodynamically relevant stenoses or occlusions of dialysis vascular access $[50,51]$. Moreover, flow-limiting stenoses can be detected also in the case of forearm fistulas with moderate sensitivity and high specificity [52]. The detection rate of central venous stenoses depends on the type of examina-
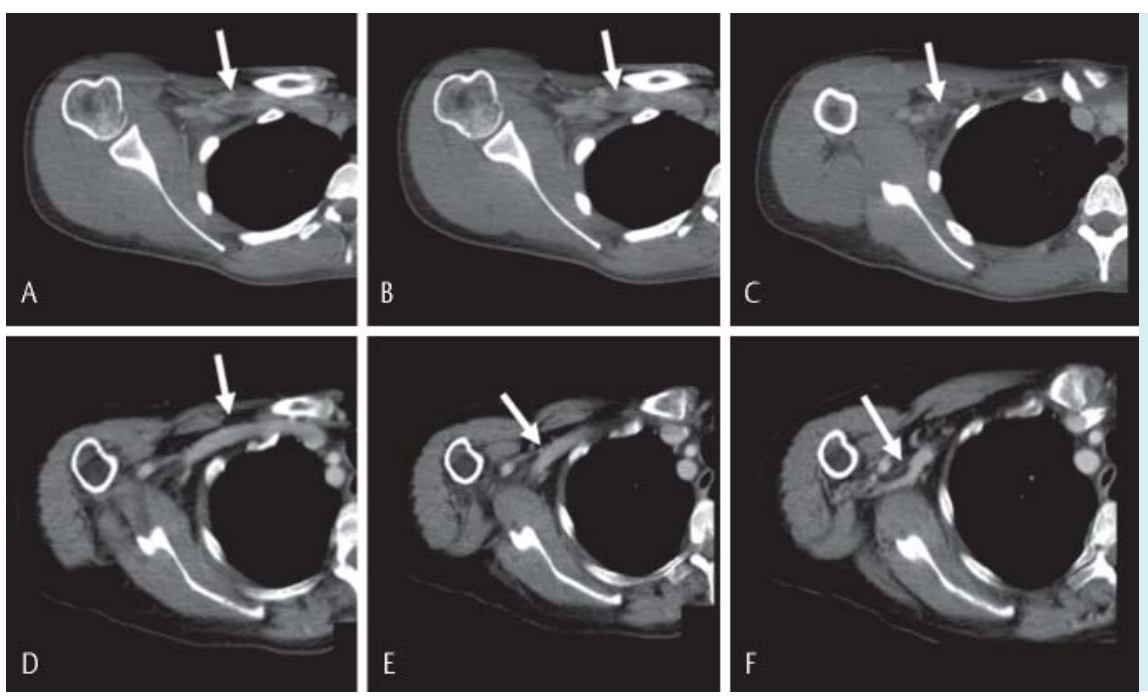

Fig. 2 Indirect $\mathrm{CT}$ venography after contralateral injection of $90 \mathrm{ml}$ lomeprol and a delay of $100 \mathrm{sec}-$ onds demonstrates central venous thrombosis (arrow in $\mathbf{A - C}$ ), compared to normal blood flow in the axillary and subclavian vein (arrow in $\mathbf{D}-\mathbf{F}$ ). 
tion protocol and CT scanner used. Karadeli et al. postulate that this method should only be taken into consideration if ultrasound examination or DSA does not provide a clear and conclusive result [47]. Current studies show that combined examinations via modern ultrasound and CT devices have comparable diagnostic reliability to that of DSA [53]. In regard to the required contrast agent quantity and radiation exposure, angiography is superior to $\mathrm{CT}$ in the case of compliance with the above conditions.

\section{Magnetic resonance imaging (MRI)}

Gadolinium-enhanced MRI can trigger nephrogenic systemic fibrosis (NSF) in patients with severe or terminally limited renal function [54]. Limited renal function is specified as the most important patient factor for the development of NSF in the guidelines of the European Society for Urogenital Radiology [55]. However, in the case of only moderate limitation of renal function, the use of contrast agent containing gadolinium with a low or moderate NSF risk can be considered [44]. Adaptation of the contrast agent dose and protocol results in a reduced incidence of new NSF cases even in dialysis patients [56].

In a French study regarding the evaluation of the incidence of NSF in 571 dialysis patients, half of whom had received macrocyclic gadolinium-based contrast agent, no new disease was seen in the observation period of four months [57]. After gadolinium-enhanced MRI, Becker and colleagues found histologically verified NSF in 2 of 49 dialysis patients [58]. However, both patients had received linear gadolinium complexes with a high NSF risk. Therefore, gadolinium-enhanced MRI examination should still not be used in dialysis patients in particular if methods equivalent or superior to sonography and angiography are available for clarifying a vascular access insufficiency [44]. If MRI examination is clinically indicated for another issue, only highly stable macrocyclic gadolinium complexes with a low NSF risk should be used at the lowest possible dose in dialysis patients [59]. Long-term data with large patient collectives regarding the specific NSF risk in dialysis patients is currently not available.

To prevent NSF risk in dialysis patients, there are some studies with unenhanced MRI examinations without gadolinium. These were able to show that preoperative vessel mapping via unenhanced MRI is technically possible [60]. However, these methods with complex sequences are still far from being able to be used in the clinical routine. To the authors' knowledge, there is currently no valid data for the evaluation of insufficient dialysis vascular access via unenhanced MRI.

\section{Interventional treatment}

In dialysis patients, a reduction of vascular diameter of more than $50 \%$ with a significant flow reduction of the ipsilateral fistula during dialysis and vascular access thrombosis are indications for treatment.

The selection of the adequate percutaneous access and the treatment method depends not only on the location, morphology, and extent of the pathology to be treated but also on the availability of an interventional radiologist with corresponding experience. Contrast agent injection via the bra-
Table 1 Possible complications of endovascular therapy with rate of occurrence modified from Haage \& Vorwerk 2010 [64].

\begin{tabular}{|c|c|}
\hline possible complication & frequency \\
\hline $\begin{array}{l}\text { venous rupture (usually able to be } \\
\text { treated endovascularly) }\end{array}$ & $\sim 8 \%$ \\
\hline $\begin{array}{l}\text { vessel rupture with loss of vascu- } \\
\text { lar access and with need for surgi- } \\
\text { cal intervention }\end{array}$ & $0.3-2 \%$ \\
\hline $\begin{array}{l}\text { pseudoaneurysm in region of the } \\
\text { puncture with need for surgical } \\
\text { intervention }\end{array}$ & $<0.5 \%$ \\
\hline $\begin{array}{l}\text { pronounced hematoma with need } \\
\text { for surgical intervention }\end{array}$ & $<0.2 \%$ \\
\hline arterial embolism & $\begin{array}{l}\text { very rare but in } 2-6 \% \text { of throm- } \\
\text { bosed brachiocephalic AV fistulas } \\
\text { in the upper arm }\end{array}$ \\
\hline $\begin{array}{l}\text { symptomatic pulmonary artery } \\
\text { embolism }\end{array}$ & $<1 \%$ \\
\hline $\begin{array}{l}\text { mesenteric ischemia, cutaneous } \\
\text { contrast agent reaction, meta- } \\
\text { bolic acidosis, pulmonary edema }\end{array}$ & $<0.1 \%$ in each case \\
\hline Infection or bacteremia & $<0.5 \%$ \\
\hline
\end{tabular}

chial artery after retrograde fine-needle puncture in the Seldinger technique should be used to visualize the total vascular access anatomy including the venous outflow. Given a known location and stenosis morphology or thrombus extent, the intervention catheter is inserted via an introducer directly so that it allows optimum access to the region to be treated antegrade or retrograde to the flow direction. Slight overdilation (by approximately $10-20 \%$ ) is recommended for treatment via percutaneous angioplasty (PTA). In the case of minimal dilation, there are increased recurrent stenoses with correspondingly lower long-term patency [61]. There are possible indications for additional stent implantation in the case of an insufficient PTA result and central venous stenoses and in the case of complications such as dissections or vascular perforations [62, 63]. In total, endovascular treatment complications in the case of dialysis vascular access are rare [64]. 0 Table 1 shows a list of possible complications.

\section{Insufficient AV fistula}

\section{Stenosis of the anastomosis}

There is a treatment indication in the case of a stenosis diameter of greater than $50 \%$ with a consecutive flow reduction, reduced dialysis rate, or previous thrombosis [21]. Stenoses of AV fistulas between the radial artery and cephalic vein (Brescia-Cimino vascular access) are primarily located in the region of the anastomosis and in the further course of the vascular access vein $[4,5]$. In the case of AV fistulas in the distal forearm, anastomosis stenoses can be treated via surgical proximalization [21]. PTA shows slightly worse long-term results and is used as an alternative method (৫ Fig.3). However, primary endovascular treatment via PTA is indicated for the proximal forearm. Stenosis of the arterial inflow - more than $2 \mathrm{~cm}$ from the anastomosis - is rare but can decrease the flow rate within the AV fistula and thus result in insufficiency of the dialysis vascular access. Stenoses of the arterial inflow should also be treated via primary PTA [21]. 

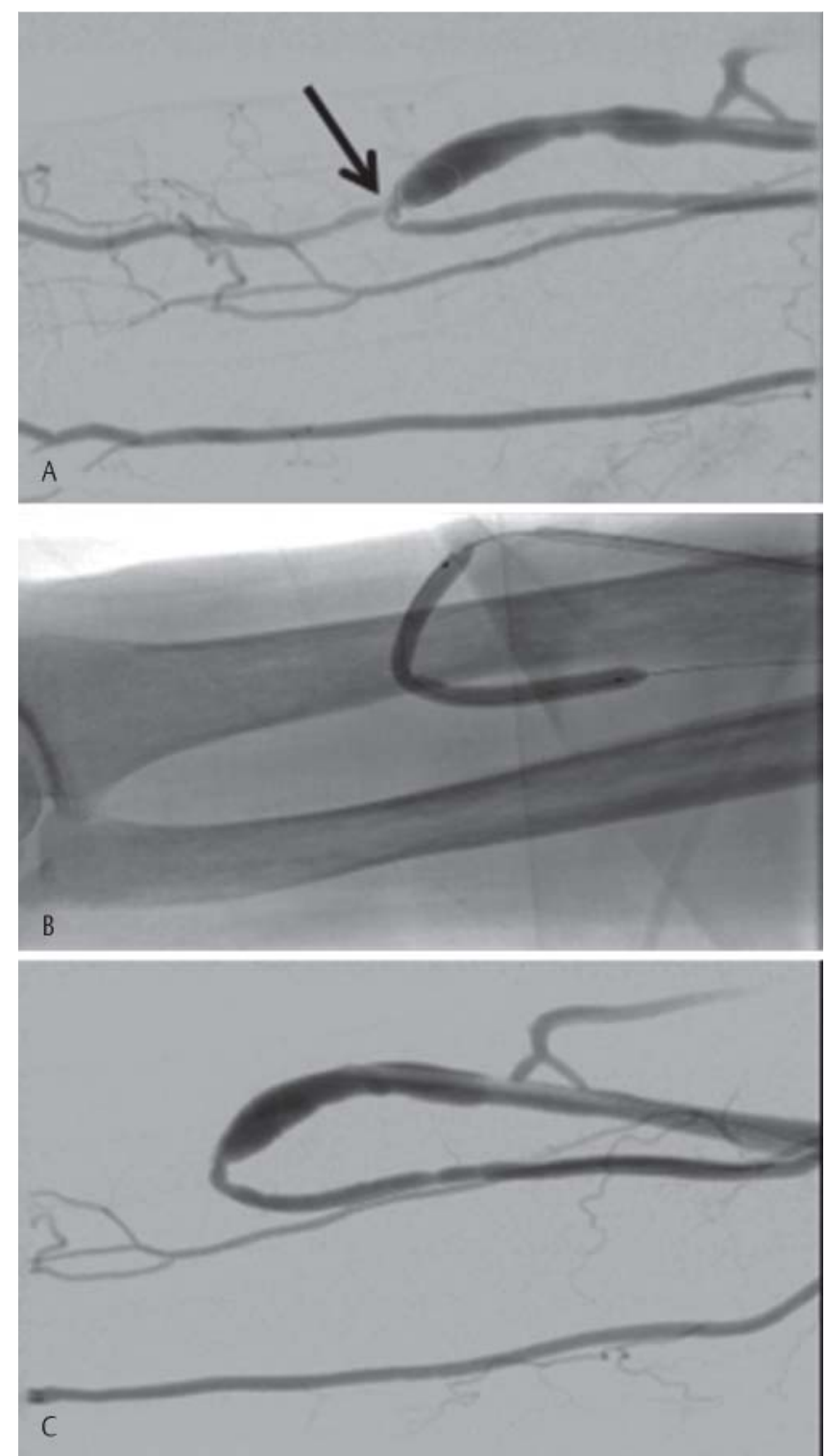

Fig. 3 Stenotic anastomosis (arrow) in a patient with radiocephalic AV fistula A. Uncomplicated PTA B with good postinterventional result C.

For stenoses near the anastomosis in the case of radiocephalic AV fistulas, residual stenosis after initial PTA represents a relevant prognosis factor. In a retrospective analysis of 73 patients, Mortamais et al. compared residual stenoses with a diameter reduction of more or less than $50 \%$. In the case of residual stenosis of less than $50 \%$, the assisted primary patency rate after 12 and 24 months was $84.6 \%$ and $76.1 \%$, respectively. For patients with residual stenosis of at least $50 \%$, the corresponding values of $62.3 \%$ and $46.8 \%$ [65] were significantly lower.

Promising patency rates were described in the use of drugcoated balloons. In the case of use in stenoses near the anastomosis, Patane et al. observed primary patency rates of $96.1 \%$ after 6 months, $81.8 \%$ after 12 months, and $57.8 \%$ after 24 months. The secondary patency rate was $95.4 \%$ after 12 months and $94.7 \%$ after 24 months [13].
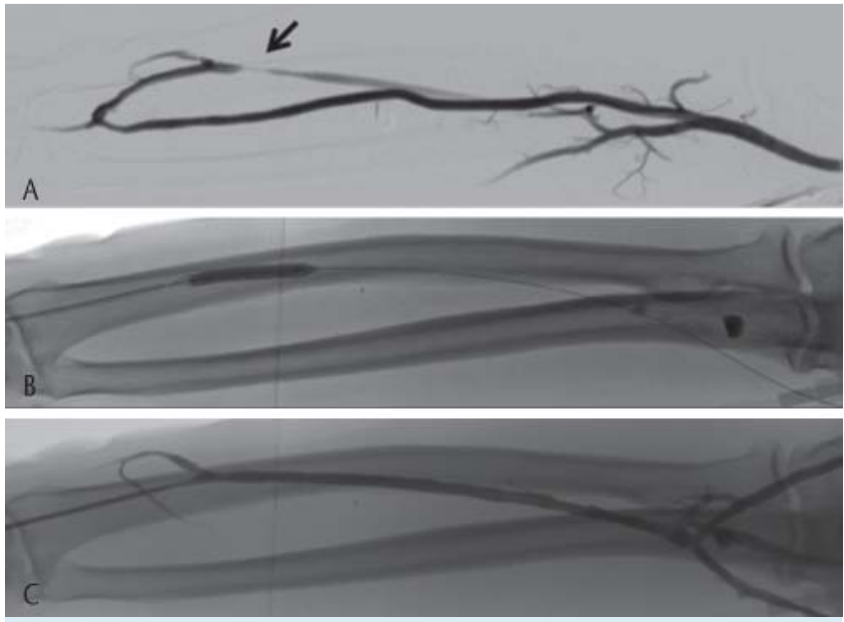

Fig. 4 Depiction of venous stenosis (arrow) in the forearm of a patient with radiocephalic AV fistula $\mathbf{A}$ after injection into the brachial artery. Uncomplicated PTA B with good result of angioplasty via an additional antegrade venous access.

Stenosis of the vascular access vein

Stenoses of the efferent arm vein in forearm fistulas are treated via primary PTA [66, 67]. Upper arm fistulas are typically stenosed in the region of the confluence of the superficial vein (typically the cephalic vein or the basilic vein) with the deep vein system [67] and should also be treated via primary PTA. In principle, vascular access vein stenoses in the forearm are accessible for interventional treatment (O Fig. 4).

Supplementary stent implantation is to be considered in the case of an insufficient PTA result and recurrent stenoses. However, stent implantation in the puncture segment should be avoided since there is no verified data regarding repeat and permanent puncture of the endoprosthesis. Overlapping of the stent into the basilic vein can prevent placement of a future brachiobasilic fistula. Interdisciplinary planning is therefore decisive for ensuring that the placement of proximal replacement vascular access is not prevented.

In a randomized study including 40 patients with stenoses in the venous branch, a higher primary patency rate $(70 \%)$ compared to conventional PTA (25\%) was observed 6 months after use of a drug-coated balloon [12]. However, there is currently no long-term data for this study.

In particular, in extremely rigid vascular access vein stenoses, the use of a cutting balloon ( $\bullet$ Fig.5) showed higher success rates compared to conventional PTA [10]. Due to the cut surfaces, overdilation results in an increased risk of perforation. Therefore, the ratio of cutting balloon to reference vessel should not be greater than 1.1 to 1 [11]. However, compared to conventional PTA, cutting balloons require a greater introducer diameter and are comparatively expensive.

When using cryoplasty catheters, reduced postinterventional neointima formation is expected due to the supplementary application of cold. As a result, lower dissection rates with similar long-term patency compared to conventional PTA in the case of arterial stenoses of the femoropopliteal circulation were described [68]. In a case series of only five patients with dialysis grafts, the superiority of 

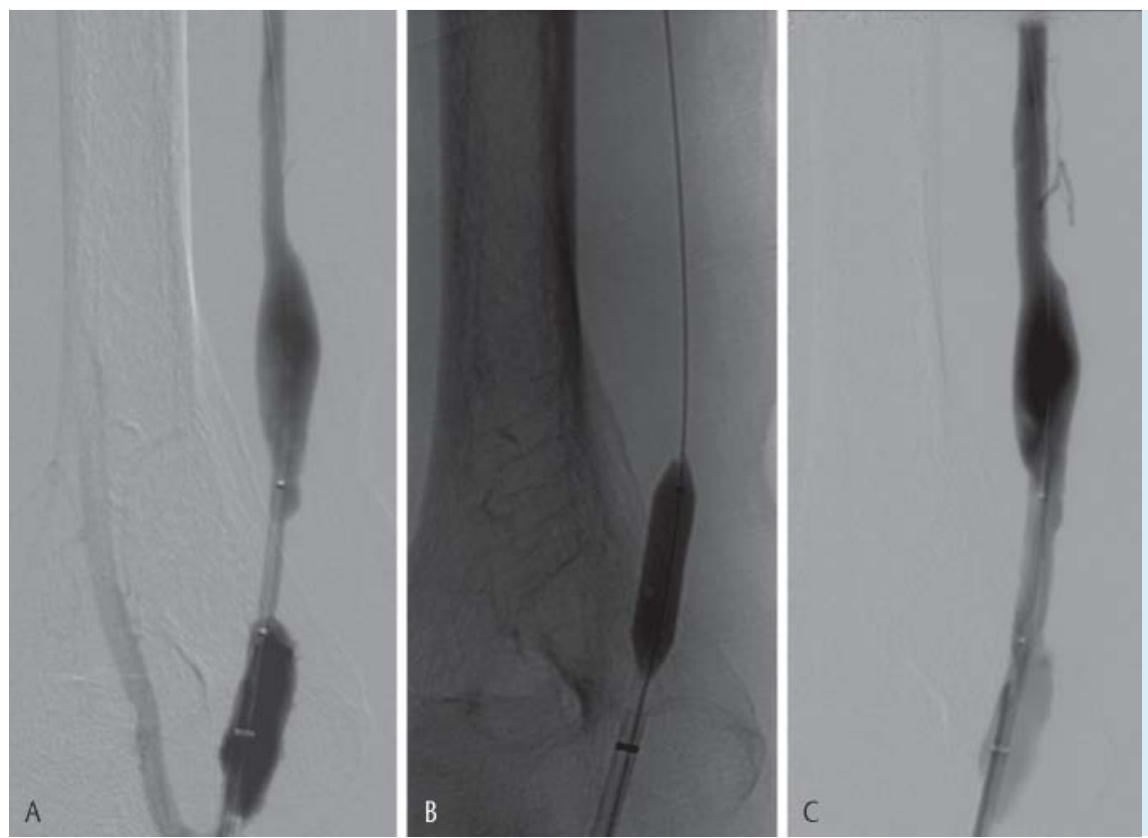

Fig. 5 Extremely rigid stenosis of the vein near the anastomosis $\mathbf{A}$ was successfully dilated $\mathbf{C}$ by a Cutting balloon $\mathbf{B}$.

cryoplasty compared to conventional PTA could be shown in the case of pronounced recurrent stenoses of the venous anastomosis [16]. However, the use of cryoplasty catheters has not yet become established. Moreover, there are no randomized studies that could confirm their superiority.

Endovascular brachytherapy and external radiation are additional interventional methods that have not yet been able to be proven to be superior and are thus not used in the clinical routine [69]. With the goal of preventing neointimal hyperplasia, brachytherapy was examined in a pilot study including 25 patients [15].

For ultrasound-guided PTA, there is currently only an insufficient number of studies of insufficient dialysis vascular access $[70,71]$.

\section{Thrombosed AV fistula}

In the case of thrombosis of the AV fistula, the age, position, and extent of the thrombosis determine the primary treatment success and the long-term patency. Treatment should ideally be initiated within 48 hours. As a result of early revascularization, placement of an alternative vascular access, such as a temporary dialysis catheter, can be prevented. Primarily urokinase and rt-PA are used as fibrinolytic agents. Accompanying mechanical thrombus manipulation via external vascular access massage or endovascular fragmentation can increase treatment success.

A short, segmental thrombosis can be sufficiently treated via PTA alone. Extensive thromboses must typically be treated via pharmacomechanical thrombolysis and consecutive balloon angioplasty [21]. Using this combination with additional thrombus aspiration, Poulain et al. achieved a one-year patency of $90 \%$ in 14 thrombosed AV fistulas [72]. Zaleski et al. treated 17 patients with thrombosed Brescia-Cimino fistulas via PTA and urokinase infusion. After a year, $71 \%$ of the AV fistulas were still patent [73]. Turmel-Rodrigues et al. describe an initial success rate of $81 \%$ in 16 patients as a result of the combined use of thrombus aspiration and PTA [74]. To conduct thrombolysis, the catheter tip (e.g. 5F catheter) should be placed within the thrombosis. After administration of a bolus of 100000 to $200000 \mathrm{IU}$ urokinase, 100000 to $200000 \mathrm{IU}$ urokinase/h should be infused over a period of 6 to 20 hours with a maximum amount of approx. 2000000 IU urokinase. In addition, approx. $5000 \mathrm{IU}$ heparin should be administered during the intervention.

As a result of thrombus fragmentation via conventional PTA, Liang and colleagues achieved a direct success rate of $93 \%$ with a patency rate of $70 \%$ after one year [75]. Residual thromboses were additionally treated via urokinase.

To date, there are no randomized studies proving the superiority of mechanical thrombectomy instruments over conventional mechanically supported pharmacological thrombolysis. The percutaneous thrombolytic device (PTD), a wire-guided instrument for mechanical thrombolysis, is mentioned as an example. The catheter is comprised of two components, the outer protective cover and an inner self-expanding fragmentation basket that is operated via the battery motor. After placement in the thrombosed vessel, the fragmentation basket is expanded. Quick rotation thereof results in mechanical thrombolysis [17].

In addition to mechanical thrombus fragmentation, hydrodynamic thrombectomy represents a further percutaneous method. In a prospective study of the angio-jet system including 187 patients with 261 dialysis grafts and 24 AV fistulas, an assisted primary patency rate after $1,6,12$, and 18 months of $72.4 \%, 45.1 \%, 30.3 \%$, and $22.4 \%$, respectively, was described [18].

In a retrospective comparison between PTD and angio-jet in 275 thrombosed AV fistulas, Yang et al. observed slightly more time-efficient thrombectomy via PTD. The primary patency rates after 6 months were $45 \%$ with the use of the angio-jet and $43 \%$ after PTD. The secondary patency rates after a year were $74 \%$ and $87 \%$, respectively [76].

In total, the treatment of the underlying stenosis determines the long-term success of thrombectomy or thrombolysis. However, this is often first visible after thrombosis treatment and should then be treated directly at the same time. 


\section{Insufficient dialysis graft}

Lumen reduction of more than $50 \%$ with significant flow reduction is a recommended indication for treatment even in dialysis grafts [21]. The most common cause of an insufficient dialysis graft is stenosis of the venous anastomosis [77]. Dialysis grafts are used in patients with "worn out" peripheral veins. Therefore, vessel-preserving endovascular methods should be primarily used in the case of graft insufficiency. Stenosis of the arterial inflow can also be effectively treated via PTA [21]. Several workgroups even observed success rates of $98 \%$ [78]. PTA can be performed without complication if only the afferent artery proximal to the vascular access or the anastomosis is affected but the artery distal to the anastomosis is not stenosed. If the efferent artery also has a distal stenosis, simultaneous dilation is recommended. PTA of the stenosed anastomosis would otherwise increase the risk of peripheral ischemia due to the steal effect as a result of the increased vascular access volume. Stenoses within the graft occur mainly in the puncture region. They are the result of connective tissue growing into the puncture tracts and can be successfully treated via PTA [21].

Supplementary stent implantation should be taken into consideration in recurrent stenoses [62]. In the case of repeat stent occlusion, it is necessary to surgically place the graft again.

A number of current studies describe improved patency rates compared to PTA alone for stenoses of the dialysis graft in the region of the venous anastomosis and the venous outflow when using covered stent grafts $[79,80]$. The study by Haskal et al. is a prospective randomized multicenter study with only a six-month follow-up [80]. A significantly lower re-stenosis rate of $28 \%$ compared to PTA with $78 \%$ after stent graft is described.

Karnabatidis et al. compare the patency rates in 35 patients after previous PTA or bare metal stent implantation with the results after a revision procedure with a covered stent graft [79]. Significantly improved primary patency rates of $61.4 \%$ compared to $8.6 \%$ after conventional PTA were observed a year after the use of a stent graft.

In the not yet published REVISE study (e.g.: http://www. accessdata.fda.gov/cdrh_docs/pdf13 / P130 006b.pdf), the treatment of stenoses of the venous anastomosis via covered stent graft and PTA alone was compared. The results showed at least no significant superiority of covered stent prostheses. Primary patency rates of $43.4 \%$ after 6 months, $21.4 \%$ after 12 months, and $9.6 \%$ after 24 months were observed for the use of stent grafts. After the use of PTA alone, the corresponding primary patency rates were $29.4 \%$, $15.2 \%$, and $6.8 \%$, respectively.

The secondary patency rates were $91.2 \%$ after 6 months, $82.7 \%$ after 12 months, and $68.9 \%$ after 24 months in patients with stent grafts and $86.5 \%, 78.8 \%$, and $66.6 \%$, respectively, after PTA alone.

Graft thromboses should be treated before the next dialysis if possible [81]. Kakisis et al. examined the use of nitinol stents in the region of venous anastomosis in patients with thrombosed brachio-axillary vascular access grafts after surgical thrombectomy. They observed significantly improved assisted primary and secondary patency rates compared to conventional PTA [82].
Older thromboses of the vascular wall (more than 5 days old) are often located behind the venous anastomosis and are difficult to access via surgical resection. Similar to thrombosed AV fistulas, thromboses of PTFE grafts can also be treated with a number of different percutaneous techniques. Thrombus aspiration, thrombolysis via fibrinolytic agents (see above) or mechanical thrombectomy can be used in combination. The treatment results of thromboses and associated stenoses of synthetic dialysis grafts were summarized by Aruny and colleagues [83]. The primary success rates of thrombolysis and mechanical thrombectomy were between $75 \%$ and $94 \%$. After 6 months, the patency was still $18-39 \%$. The patency rates 6 and 12 months after thrombolysis were $62-80 \%$ and $57-69 \%$, respectively. Trerotola and colleagues demonstrated a $95 \%$ primary success rate with a patency rate after 3 months of $39 \%$ as a result of the use of PTD [84]. Compared to different mechanical thrombolysis instruments, Smits et al. concluded that the treatment of the underlying stenosis was the only relevant factor for the treatment success of a thrombosed dialysis graft [85]. Therefore, the method should be selected as a function of the experience of the particular interventional radiology center.

\section{Central venous stenoses and occlusions}

In addition to the superior vena cava, the brachiocephalic vein, and the subclavian vein, the opening region of the cephalic vein is also included as a central venous vessel ( $\bullet$ Fig.6). Due to the accelerated flow rates in the venous
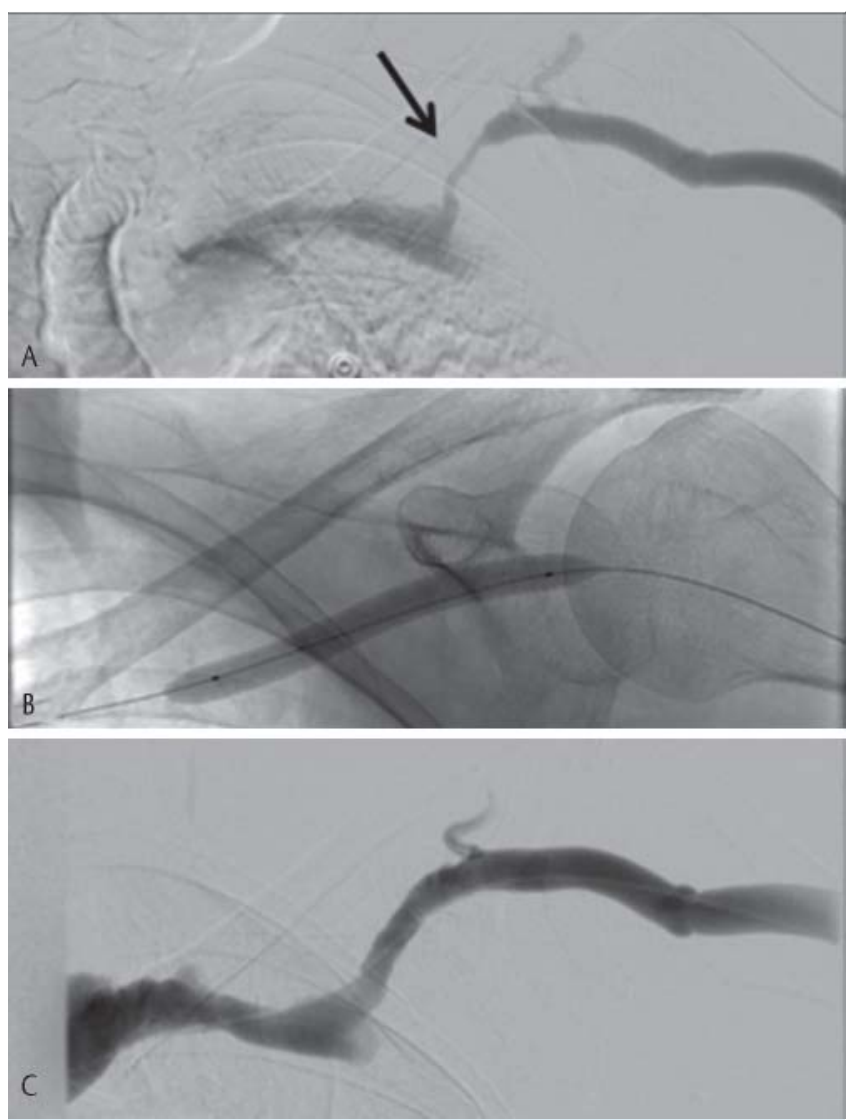

Fig. 6 Cephalic arch stenosis $\mathbf{A}$ with uncomplicated balloon dilation $\mathbf{B}$ and good PTA result. 

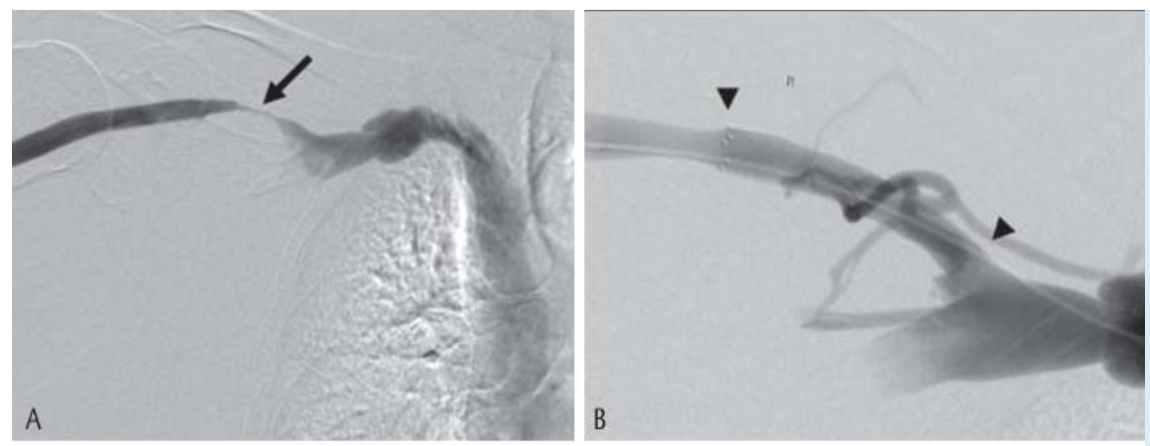

Fig. 7 Right-sided cephalic arch stenosis $\mathbf{A}$ with uncomplicated stent implantation due to insufficient PTA B. In-stent stenosis was diagnosed three years after stent implantation $\mathbf{C}$ and was successfully treated by PTA $\mathbf{D}$.
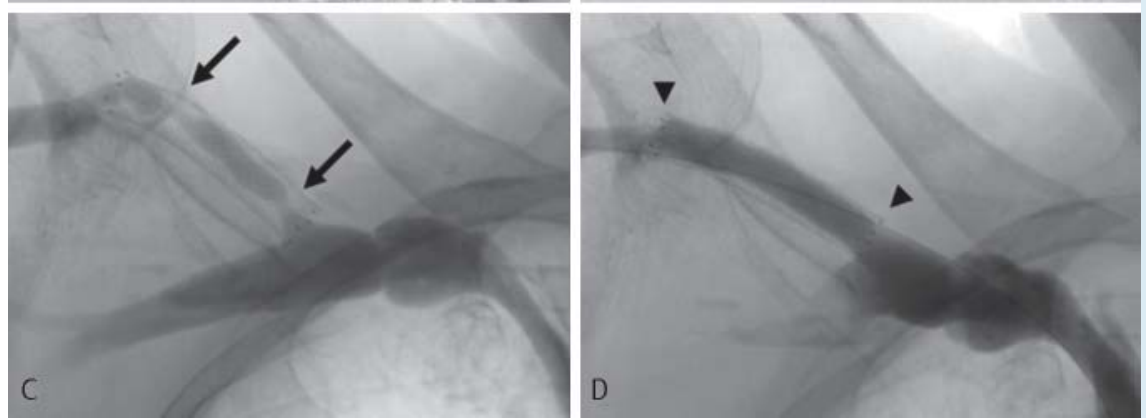

branch, dialysis patients develop rigid central venous stenoses in $11-50 \%$ of cases [6]. Previous trauma to the venous wall caused by central venous dialysis catheters or flow obstructions caused by port catheters or pacemaker electrodes represent additional risk factors $[6,86]$. Therapy is indicated in the case of symptomatic central venous stenosis with painful swelling of the arms, restricted movement, or insufficient hemodialysis. Antegrade puncture of the vascular access vein is performed for interventional therapy. The balloon diameters to be used for PTA are selected in relation to the non-stenosed vessel segment. Careful dilation of the vasoconstriction to the caliber of the healthy vessel segment is recommended [6]. In the case of dialysis patients, central stenoses in the opening region of the cephalic vein and the brachiocephalic vein are described $[87,88]$. Both regions are accessible for percutaneous intervention via the antegrade venous access. Alternatively, femoral-venous access is possible in the case of impassable brachial stenoses or occlusions. If a treatable central venous stenosis or occlusion is identified as the cause of the central flow disturbance, treatment with PTA is recommended. In the case of untreatable malignant compression or pacemaker electrodes, there is an indication for primary stent implantation [21]. A stent in the region of a venous confluence should be avoided if possible since a consecutive stenosis can occur in the case of overlapping into the non-stenosed vessel. Therefore, for example, a stent in the confluence of the cephalic vein and the subclavian vein can induce stenosis and prevent venous drainage of the arm which would then prevent subsequent placement of a brachial AV fistula. A subclavian stent with overlapping into the opening of the jugular vein can complicate the cervical placement of a temporary dialysis catheter. In-stent recurrent stenoses ( $\bullet$ Fig. 7) can be treated via repetitive PTA.

Both PTA and stent implantation are effective and reliable methods for central venous stenoses. Ozyer and colleagues observed a comparable complication rate after stent implantation and PTA alone in a single-center analysis of 126 patients with central venous stenoses. However, a significantly higher re-intervention rate was seen after stent implantation and significantly higher primary patency rates after PTA alone [89]. Due to the necessity for repeat treatment of relapses, avoidance of central venous dialysis catheters as a prophylaxis for central venous obstructions continues to be recommended [21]. In patients with central recurrent stenoses of the cephalic vein, superiority of covered stent grafts compared to normal bare stents in relation to the patency rate was able to be shown [90]. However, these results are a subject of controversy since the placement of a covered stent could induce central stenoses and occlusions that would prevent future placement of an ipsilateral vascular access [91].

In the case of a central venous occlusion ( $\bullet$ Fig. 8 ), there is a significant increase in the volume of the ipsilateral arm [92]. Moreover, prominence of the superficial arm veins due to the collateral flow, paresthesia, and pain are observed. The treatment of central venous occlusions and of in-stent thromboses ( $\bullet$ Fig.9) can also be performed via PTA or primary stent implantation [21]. However, dialysis can remain possible in the case of a sufficient collateral system with a corresponding flow volume despite central venous occlusion.

After treatment, frequent clinical monitoring should be performed. The occurrence of pain or swelling as well as a flow reduction during dialysis are an indication for performing clarification again.

Complications such as pulmonary artery embolism, vascular wall damage, and perforation are extremely rare ( $\bullet \mathrm{Ta}$ ble 1). However, vascular wall rupture of a central vein can be associated with life-threatening pericardial tamponade or a large pleural effusion. In the case of the slightest indication of a tear in the wall of a central vein, the PTA balloon should be left near the lesion to be able to inflate it again quickly as needed to cover the site of the perforation. Implantation of a bare metal stent can treat smaller damage 

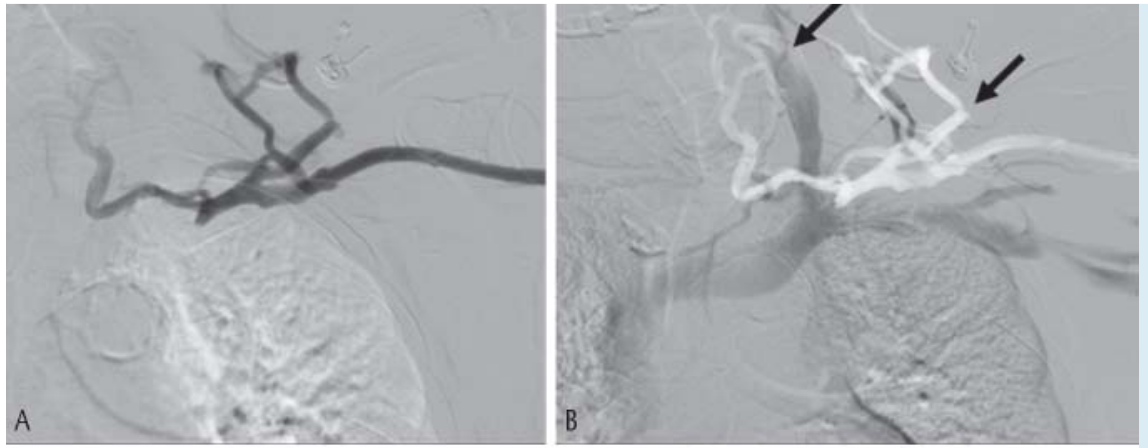

Fig. 8 Unrecanalizable central venous occlusion by brachial approach $\mathbf{A}$ with delayed outflow via collateral vessels (arrows in B). Additional visualization of the occlusion via femoral access $\mathbf{C}$. Simultaneous injection displayed the complete length of the occlusion $\mathbf{D}$, but the occlusion was still not recanalizable via the femoral approach.
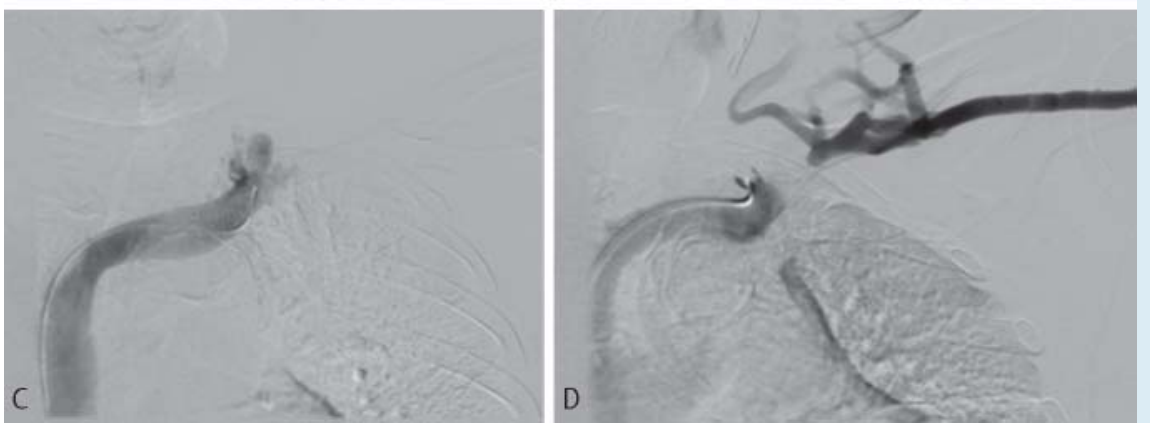
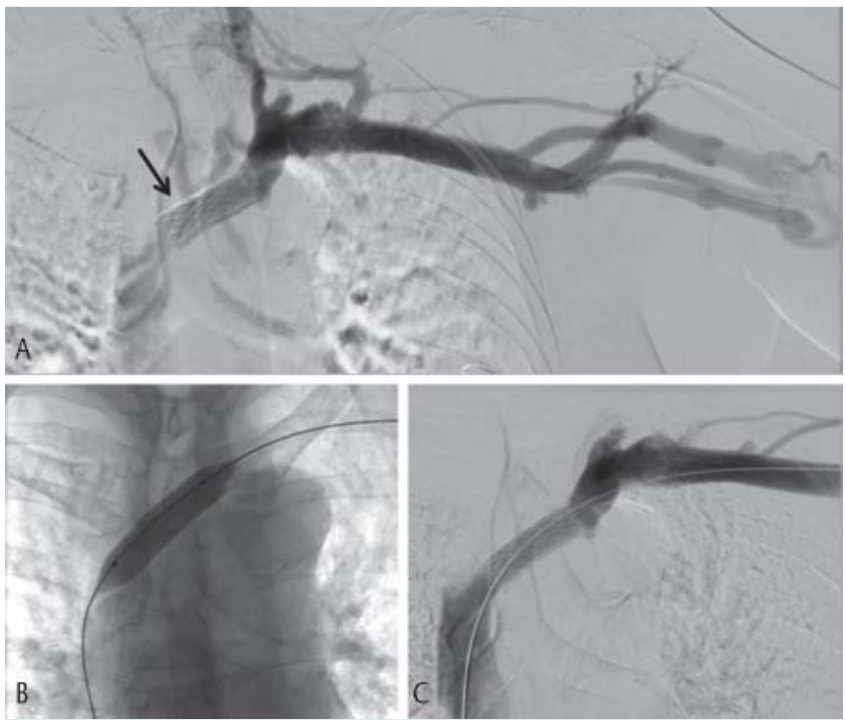

Fig. 9 Angiographic diagnosis of a stent occlusion 6 months after implantation in the left brachiocephalic vein A. Uncomplicated recanalization by PTA B. Post-interventional angiography showed a significant reduction of collateral vessels $\mathbf{C}$.

to the vascular wall. However, implantation of a covered stent is recommended in the case of a perforation.

\section{Periinterventional medication}

The use of anticoagulants in the treatment of central venous lesions pre-, peri-, and postinterventionally is not confirmed by large studies. The following data are thus to be viewed as recommendations.

After placement of the introducer, a bolus of 3000 to 5000 IU unfractionated heparin is administered intravenously before intraluminal treatment [86, 93]. After stent implantation, PTT-relevant intravenous administration of up to 1000 IU heparin per hour for 24 hours can be consid- ered. With respect to the administration of low-molecular heparin (e.g. enoxaparin), the manufacturer specifications for dose adaptation and monitoring should be taken into consideration particularly in patients with limited renal function to avoid bleeding complications.

The effectiveness of thrombocyte function inhibition via medication in venous intraluminal treatments is not proven in contrast to the arterial vascular system. However, it is conceivable that there is an advantage based on the arterialized flow. Oral application of acetylsalicylic acid has been propagated for over a decade. The administration of clopidogrel at $75 \mathrm{mg}$ per day for 4 to 6 weeks after stent implantation currently seems advisable.

\section{Discussion}

$\nabla$

At present, the number of patients with chronic renal insufficiency continues to increase as a result of continuously improving treatment methods and a decreasing mortality rate. The dysfunction of arteriovenous fistulas is a main cause for the high morbidity and hospitalization rate (20$30 \%$ ) of hemodialysis patients. Despite all improvements in the care of this patient collective, only approximately $15 \%$ of ateriovenous fistulas remain functional over the long term. As a result, the diagnosis and treatment of vascular access dysfunction in hemodialysis patients are extremely important and are a central point in treatment.

Unfortunately, the recommendations in all guidelines of the various associations and organizations regarding the improvement of the treatment of ESRD (end-stage renal disease) must relate to publications with evidence levels 2 and 3, far removed from level 1 randomized studies. Nonetheless, internationally accepted and recognized diagnostic and therapeutic procedures can be derived from these available guidelines and from evidence collected by many workgroups over decades and these are described in this article. 
Table 2 List of possible pathological findings in vascular access insufficiency with corresponding treatment indications and different treatment options.

\begin{tabular}{|c|c|c|c|}
\hline & pathology & treatment indication & treatment modality \\
\hline \multirow[t]{4}{*}{ AV fistula } & anastomosis stenosis & $\begin{array}{l}>50 \% \text { stenosis with flow reduction, } \\
\text { insufficient dialysis, or thrombosis }\end{array}$ & $\begin{array}{l}\text { - endovascular treatment attempt with PTA } \\
\text { - surgical treatment via proximalization of } \\
\text { anastomosis in case of treatment failures }\end{array}$ \\
\hline & $\begin{array}{l}\text { venous stenosis near } \\
\text { anastomosis }\end{array}$ & $\begin{array}{l}>50 \% \text { stenosis with flow reduction, } \\
\text { insufficient dialysis, or thrombosis }\end{array}$ & $\begin{array}{l}\text { - endovascular treatment attempt with PTA } \\
\text { - surgical treatment via proximalization of } \\
\text { anastomosis }\end{array}$ \\
\hline & $\begin{array}{l}\text { venous stenosis of the efferent } \\
\text { vein }\end{array}$ & $\begin{array}{l}>50 \% \text { stenosis with flow reduction, } \\
\text { insufficient dialysis, or thrombosis }\end{array}$ & $\begin{array}{l}\text { - primary endovascular treatment with PTA } \\
\text { - stent implantation in case of an insufficient } \\
\text { result, complications, or early relapse } \\
\text { (<3 months) }\end{array}$ \\
\hline & thrombosis of the AV fistula & insufficient dialysis & $\begin{array}{l}\text { dependent on age, location, and extent of } \\
\text { thrombosis } \\
\text { - short segmental thrombosis with PTA } \\
\text { - longer thrombosis via endovascular throm- } \\
\text { bectomy }\end{array}$ \\
\hline \multirow[t]{3}{*}{ Dialysis graft } & stenosis & $\begin{array}{l}>50 \% \text { stenosis with flow reduction, } \\
\text { insufficient dialysis, or thrombosis }\end{array}$ & $\begin{array}{l}\text { - primary endovascular treatment with PTA } \\
\text { - stent implantation in case of an insufficient } \\
\text { result, complications, or early relapse } \\
\text { (<3 months) }\end{array}$ \\
\hline & stenosis of venous anastomosis & $\begin{array}{l}>50 \% \text { stenosis with flow reduction, } \\
\text { insufficient dialysis, or thrombosis }\end{array}$ & - primary implantation of a stent graft \\
\hline & thrombosis & insufficient dialysis & $\begin{array}{l}\text { dependent on age, location, and extent } \\
\text { - short segmental thrombosis: PTA } \\
\text { - longer thrombosis: endovascular throm- } \\
\text { bectomy }\end{array}$ \\
\hline $\begin{array}{l}\text { Central venous ob- } \\
\text { struction }\end{array}$ & stenosis/occlusion & $\begin{array}{l}\text { painful arm swelling with loss of function } \\
>50 \% \text { stenosis with flow reduction, } \\
\text { insufficient dialysis, or thrombosis }\end{array}$ & $\begin{array}{l}\text { - primary endovascular treatment with PTA via } \\
\text { brachial access } \\
\text { - femoral access in case of impassable stenosis } \\
\text { - stent implantation in case of an insufficient } \\
\text { result, complications, or early relapse } \\
\text { (<3 months) }\end{array}$ \\
\hline
\end{tabular}

This includes a collaboration of interventional radiologists, vascular surgeons, and nephrologists which is the starting point for sufficient diagnostics and indication determination in the treatment of insufficient dialysis vascular access ( Table 2). Diagnosis is made via a combination of clinical symptoms, duplex sonography, and supplementary angiography in the case of expected intervention.

Compared to ultrasound, DSA allows better evaluation of the central veins. Moreover, it is superior to CT with respect to the required contrast agent quantity. In the diagnosis of a vascular access insufficiency, CT is currently still a secondary method. However, in the case of suspected mediastinal vascular compression, diagnosis via sectional imaging is essential.

Brachial stenoses of the efferent vascular access veins and central venous stenoses should be primarily treated via PTA or in combination with stent implantation after careful consideration in the case of complication or an insufficient PTA result. To date, no stent type has proven to be superior among uncoated bare metal stents [89]. The use of cutting balloons can be considered in the case of extremely rigid or elastic stenoses. With respect to the re-stenosis rate, drugcoated balloons show promising results. However, studies with larger patient collectives and long-term data are currently not available. Long-term results for stent grafts in the region of venous anastomosis of vascular access grafts are also not yet available. To date, endovascular brachytherapy and external radiation have not been able to show su- perior results and currently do not play a major role in the treatment of vascular access insufficiency.

In the treatment of vascular access thromboses, surgical and interventional thrombectomy has comparable results. Among interventional methods, purely mechanical and pharmacomechanical approaches yield comparable patency rates. Segmental thromboses should therefore be treated interventionally and extensive occlusions should be treated surgically. Regardless of the method used, subsequent PTA of the etiological stenosis is the most important prognostic factor after thrombosis treatment [85].

Central venous occlusion should be treated primarily percutaneously via PTA. Stent implantation should be discussed in the case of PTA failure due to recoil, incomplete PTA, or quick re-obstruction within three to six months. Open surgical treatment should only be considered in the case of occlusion that cannot be treated via intervention due the higher level of invasiveness. Large vascular access aneurysms should preferably be treated surgically. Endovascular treatment can be performed as an ambulant intervention. The vascular access can subsequently be used directly for dialysis.

In summary, the indication for treatment should always be made in interdisciplinary consensus and the treatment modality should be selected as a function of the local expertise. 


\section{References}

1 Gilg J, Rao A, Fogarty D. UK Renal Registry 16th Annual Report: Chapter 1 UK Renal Replacement Therapy Incidence in 2012: National and Centre-specific Analyses. Nephron Clin Pract 2013; 125: 1-27

2 Bray BD, Boyd J, Daly C et al. Vascular access type and risk of mortality in a national prospective cohort of haemodialysis patients. QJM Mon J Assoc Physicians 2012; 105: 1097-1103

3 Stolic R. Most Important Chronic Complications of Arteriovenous Fistulas for Hemodialysis. Med Princ Pract 2013; 22: 220-228

4 Mickley $V$. Stenosis and thrombosis in haemodialysis fistulae and grafts: the surgeon's point of view. Nephrol Dial Transplant 2004; 19 : 309-311

5 Sivanesan S, How TV, Bakran A. Sites of stenosis in AV fistulae for haemodialysis access. Nephrol Dial Transplant 1999; 14: 118-120

6 Mansour M, Kamper L, Altenburg A et al. Radiological central vein treatment in vascular access. J Vasc Access 2008; 9: 85-101

7 Kamper L, Haage P. Endovaskuläre Verfahren. In: Hepp W, Koch M, Konner K (Hrsg.) Dialyseshunts. 2. Aufl. Darmstadt: Steinkopff; 2009

8 Tattersall J, Martin-Malo A, Pedrini L et al. EBPG guideline on dialysis strategies. Nephrol Dial Transplant Off Publ Eur Dial Transpl Assoc Eur Ren Assoc 2007; 22: ii5-21

9 Kamper L, Frahnert M, Grebe S-O et al. Radiological assessment of vascular access in haemodialysis patients. J Vasc Access 2014; 15: 33 - 37

10 Guiu B, Loffroy R, Ben SalemD et al. Angioplasty of long venous stenoses in hemodialysis access: at last an indication for cutting balloon? J Vasc Interv Radiol 2007; 18: 994-1000

11 Vesely TM, Siegel JB. Use of the peripheral cutting balloon to treat hemodialysis-related stenoses. J Vasc Interv Radiol 2005; 16: 1593 - 1603

12 Katsanos K, Karnabatidis D, Kitrou P et al. Paclitaxel-coated balloon angioplasty vs. plain balloon dilation for the treatment of failing dialysis access: 6-month interim results from a prospective randomized controlled trial. J Endovasc Ther Off J Int Soc Endovasc Spec 2012; 19: $263-272$

13 Patanè D, Giuffrida S, Morale W et al. Drug-eluting balloon for the treatment of failing hemodialytic radiocephalic arteriovenous fistulas: our experience in the treatment of juxta-anastomotic stenoses. J Vasc Access 2012; DOI: 10.5301/jva.5000211

14 Ahmed S, Roy-Chaudhury P. Radiation Therapy for Dialysis Access Stenosis: Unfulfilled Promise or False Expectations. Semin Dial 2012; 25: $464-469$

15 Misra S, Bonan R, Pflederer T et al. BRAVO I: A pilot study of vascular brachytherapy in polytetrafluoroethylene dialysis access grafts. Kidney Int 2006; 70: 2006-2013

16 Rifkin BS, Brewster UC, Aruny JE et al. Percutaneous balloon cryoplasty: a new therapy for rapidly recurrent anastomotic venous stenoses of hemodialysis grafts? Am J Kidney Dis Off J Natl Kidney Found 2005; 45: e27-32

17 Truong TH, Spuentrup E, Staatz G et al. Mechanical thrombectomy of iliocaval thrombosis using a protective expandable sheath. Cardiovasc Intervent Radiol 2004; 27: 254-258

18 Kakkos SK, Haddad GK, Haddad J et al. Percutaneous rheolytic thrombectomy for thrombosed autogenous fistulae and prosthetic arteriovenous grafts: outcome after aggressive surveillance and endovascular management. J Endovasc Ther Off J Int Soc Endovasc Spec 2008; 15: $91-102$

19 Lee T, Mokrzycki M, Moist L et al. Standardized definitions for hemodialysis vascular access. Semin Dial 2011; 24: 515-524

20 Sidawy AN. , Society for Vascular Surgery. Arteriovenous hemodialysis access: the Society for Vascular Surgery practice guidelines. J Vasc Surg 2008; 48: $1 \mathrm{~S}$

21 Tordoir J, Canaud B, Haage P et al. EBPG on Vascular Access. Nephrol Dial Transplant Off Publ Eur Dial Transpl Assoc - Eur Ren Assoc 2007; 22: ii $88-117$

22 Vascular Access Work Group. Clinical practice guidelines for vascular access. Am J Kidney Dis Off J Natl Kidney Found 2006; 48: S248 -S273

23 III. NKF-K/DOQI Clinical Practice Guidelines for Vascular Access: update 2000. Am J Kidney Dis Off J Natl Kidney Found 2001; 37: S137S181

24 Robbin ML, Chamberlain NE, Lockhart ME et al. Hemodialysis arteriovenous fistula maturity: US evaluation. Radiology 2002; 225: 59-64

25 Thalhammer C, Aschwanden M, Staub D et al. Duplex sonography of hemodialysis access. Ultraschall in Med Stuttg Ger 1980 2007; 28: 450 465, quiz 466-471

26 Doelman C, Duijm LEM, Liem YS et al. Stenosis detection in failing hemodialysis access fistulas and grafts: comparison of color Doppler ul- trasonography, contrast-enhanced magnetic resonance angiography, and digital subtraction angiography. J Vasc Surg 2005; 42: 739-746

27 Ren J, Han X, Liu $\mathrm{H}$ et al. Ultrasonographic assessment of polytetrafluoroethylene upper extremity arteriovenous hemodialysis access: a retrospective analysis of 31 cases. Ther Apher Dial Off Peer-Rev J Int Soc Apher Jpn Soc Apher Jpn Soc Dial Ther 2012; 16: 548-553

28 Tordoir JH, Hoeneveld H, Eikelboom BC et al. The correlation between clinical and duplex ultrasound parameters and the development of complications in arterio-venous fistulae for haemodialysis. Eur J Vasc Surg 1990; 4: 179-184

29 Older RA, Gizienski TA, Wilkowski MJ et al. Hemodialysis access stenosis: early detection with color Doppler US. Radiology 1998; 207: 161 164

30 Malik J, Slavikova M, Svobodova J et al. Regular ultrasonographic screening significantly prolongs patency of PTFE grafts. Kidney Int 2005; 67: $1554-1558$

31 Amin MZ, Vesely TM, Pilgram T. Correlation of intragraft blood flow with characteristics of stenoses found during diagnostic fistulography. J Vasc Interv Radiol 2004; 15: 589-593

32 Nassar GM, Ayus JC. Infectious complications of the hemodialysis access. Kidney Int 2001; 60: 1-13

33 Schwarz C, Mitterbauer C, Boczula M et al. Flow monitoring: performance characteristics of ultrasound dilution versus color Doppler ultrasound compared with fistulography. Am J Kidney Dis Off J Natl Kidney Found 2003; 42: 539-545

34 Wiese P, Nonnast-Daniel B. Colour Doppler ultrasound in dialysis access. Nephrol Dial Transplant Off Publ Eur Dial Transpl Assoc - Eur Ren Assoc 2004; 19: 1956 - 1963

35 Dumars MC, Thompson WE, Bluth EI et al. Management of suspected hemodialysis graft dysfunction: usefulness of diagnostic US. Radiology 2002; 222: $103-107$

36 Kudlicka J, Kavan J, Tuka V et al. More precise diagnosis of access stenosis: ultrasonography versus angiography. J Vasc Access 2012; 13: $310-$ 314

37 Haage $P$, Vorwerk $D$, Piroth $W$ et al. Treatment of hemodialysis-related central venous stenosis or occlusion: results of primary Wallstent placement and follow-up in 50 patients. Radiology 1999; 212: 175180

38 Duijm LEM, van der Rijt RHH, Cuypers PWM et al. Outpatient treatment of arterial inflow stenoses of dysfunctional hemodialysis access fistulas by retrograde venous access puncture and catheterization. J Vasc Surg 2008; 47: $591-598$

39 Asif A, Cherla G, Merrill D et al. Venous mapping using venography and the risk of radiocontrast-induced nephropathy. Semin Dial 2005; 18 : 239-242

40 Bucher AM, De Cecco CN, Schoepf UJ et al. Is Contrast Medium Osmolality a Causal Factor for Contrast-Induced Nephropathy? BioMed Res Int 2014; 2014: e931413

41 Wessely R, Koppara T, Bradaric $C$ et al. Choice of Contrast Medium in Patients With Impaired Renal Function Undergoing Percutaneous Coronary Intervention. Circ Cardiovasc Interv 2009; 2: 430-437

42 Heye S, Maleux G, Marchal GJ. Upper-extremity venography: CO2 versus iodinated contrast material. Radiology 2006; 241: 291-297

43 Kariya S, Tanigawa N, Kojima $\mathrm{H}$ et al. Efficacy of carbon dioxide for diagnosis and intervention in patients with failing hemodialysis access. Acta Radiol Stockh Swed 1987 2010; 51: 994-1001

44 Heverhagen J, Krombach G, Gizewski E. Application of Extracellular Gadolinium-based MRI Contrast Agents and the Risk of Nephrogenic Systemic Fibrosis. Fortschr Röntgenstr 2014; 186: 661-669

45 Lehmkuhl L, Foldyna B, Haensig $M$ et al. Role of preprocedural computed tomography in transcatheter aortic valve implantation. Fortschr Röntgenstr 2013; 185: 941 - 949

46 Ye C, Mao Z, Rong $S$ et al. Multislice computed tomographic angiography in evaluating dysfunction of the vascular access in hemodialysis patients. Nephron Clin Pract 2006; 104: c94-100

47 Karadeli E, Tarhan NC, Ulu EMK et al. Evaluation of failing hemodialysis fistulas with multidetector $\mathrm{CT}$ angiography: comparison of different 3D planes. Eur J Radiol 2009; 69: 184-192

$48 \mathrm{Ko} \mathrm{S}-\mathrm{F}$, Huang C-C, Ng S-H et al. MDCT angiography for evaluation of the complete vascular tree of hemodialysis fistulas. Am J Roentgenol 2005; 185 : $1268-1274$

49 Dimopoulou A, Raland H, Wikström B et al. MDCT angiography with 3D image reconstructions in the evaluation of failing arteriovenous fistulas and grafts in hemodialysis patients. Acta Radiol Stockh Swed 1987 2011; 52: 935-942 
50 Wasinrat J, Siriapisith T, Thamtorawat S et al. 64-slice MDCT angiography of upper extremity in assessment of native hemodialysis access. Vasc Endovascular Surg 2011; 45: 69-77

51 Heye S, Maleux G, Claes K et al. Stenosis detection in native hemodialysis fistulas with MDCT angiography. Am J Roentgenol 2009; 192: $1079-1084$

52 Rooijens PPGM, Serafino GP, Vroegindeweij D et al. Multi-slice computed tomographic angiography for stenosis detection in forearm hemodialysis arteriovenous fistulas. J Vasc Access 2008; 9: 278-284

53 Cansu A, Soyturk M, Ozturk MH et al. Diagnostic value of color Doppler ultrasonography and MDCT angiography in complications of hemodialysis fistulas and grafts. Eur J Radiol 2013; 82: 1436-1443

54 Prince MR, Zhang HL, Prowda JC et al. Nephrogenic Systemic Fibrosis and Its Impact on Abdominal Imaging1. RadioGraphics 2009; 29: $1565-1574$

55 Thomsen HS, Morcos SK, Almén T et al. Nephrogenic systemic fibrosis and gadolinium-based contrast media: updated ESUR Contrast Medium Safety Committee guidelines. Eur Radiol 2013; 23: 307-318

56 Martin DR, Krishnamoorthy SK, Kalb B et al. Decreased incidence of NSF in patients on dialysis after changing gadolinium contrast-enhanced MRI protocols. J Magn Reson Imaging 2010; 31: 440 - 446

57 Amet S, Launay-Vacher V, Clément $O$ et al. Incidence of Nephrogenic Systemic Fibrosis in Patients Undergoing Dialysis After Contrast-Enhanced Magnetic Resonance Imaging With Gadolinium-Based Contrast Agents: The Prospective Fibrose Nephrogénique Systémique Study. Invest Radiol 2014; 49: 109-115

58 Becker S, Walter S, Witzke O et al. Application of Gadolinium-Based Contrast Agents and Prevalence of Nephrogenic Systemic Fibrosis in a Cohort of End-Stage Renal Disease Patients on Hemodialysis. Nephron Clin Pract 2012; 121: c91 - c94

59 Morcos SK. Dialysis and Contrast Media. In: Thomsen HS, Webb JAW (Hrsg.). Contrast Media. Berlin Heidelberg: Springer; 2014: 105-109

60 Bode AS, Planken RN, Merkx MAG et al. Feasibility of non-contrast-enhanced magnetic resonance angiography for imaging upper extremity vasculature prior to vascular access creation. Eur J Vasc Endovasc Surg Off J Eur Soc Vasc Surg 2012; 43: 88 - 94

61 Turmel-Rodrigues L, Pengloan J, Bourquelot P. Interventional radiology in hemodialysis fistulae and grafts: a multidisciplinary approach. Cardiovasc Intervent Radiol 2002; 25: 3-16

62 Vorwerk D, Guenther RW, Mann H et al. Venous stenosis and occlusion in hemodialysis shunts: follow-up results of stent placement in 65 patients. Radiology 1995; 195: 140-146

63 Vorwerk $D$, Bücker A, Alzen G et al. Chronic venous occlusions in haemodialysis shunts: efficacy of percutaneous treatment. Nephrol Dial Transplant Off Publ Eur Dial Transpl Assoc - Eur Ren Assoc 1995; 10: $1869-1873$

64 Haage $P$, Vorwerk D. Dialysis Fistulas. In: Complex Cases Peripher. Vasc Interv. New York: Informa Healthcare; 2010: 239-256

65 Mortamais J, Papillard M, Girouin N et al. Endovascular Treatment of Juxta-anastomotic Venous Stenoses of Forearm Radiocephalic Fistulas: Long-term Results and Prognostic Factors. J Vasc Interv Radiol 2013; 24: $558-564$

66 Turmel-Rodrigues L, Pengloan J, Blanchier D et al. Insufficient dialysis shunts: improved long-term patency rates with close hemodynamic monitoring, repeated percutaneous balloon angioplasty, and stent placement. Radiology 1993; 187: 273-278

67 Turmel-Rodrigues L, Pengloan J, Baudin S et al. Treatment of stenosis and thrombosis in haemodialysis fistulas and grafts by interventional radiology. Nephrol Dial Transplant Off Publ Eur Dial Transpl Assoc Eur Ren Assoc 2000; 15: 2029-2036

68 Laird J, Jaff MR, Biamino G et al. Cryoplasty for the treatment of femoropopliteal arterial disease: results of a prospective, multicenter registry. J Vasc Interv Radiol 2005; 16: 1067-1073

69 Cohen GS, Freeman H, Ringold MA et al. External beam irradiation as an adjunctive treatment in failing dialysis shunts. J Vasc Interv Radiol 2000; 11: $321-326$

70 Gorin DR, Perrino L, Potter DM et al. Ultrasound-guided angioplasty of autogenous arteriovenous fistulas in the office setting. J Vasc Surg 2012; 55: $1701-1705$

71 Ascher E, Hingorani A, Marks N. Duplex-guided balloon angioplasty of failing or nonmaturing arterio-venous fistulae for hemodialysis: a new office-based procedure. J Vasc Surg 2009; 50: 594-599

72 Poulain F, Raynaud A, Bourquelot Pet al. Local thrombolysis and thromboaspiration in the treatment of acutely thrombosed arteriovenous hemodialysis fistulas. Cardiovasc Intervent Radiol 1991; 14: 98-101
73 Zaleski GX, Funaki B, Kenney S et al. Angioplasty and bolus urokinase infusion for the restoration of function in thrombosed Brescia-Cimino dialysis fistulas. J Vasc Interv Radiol 1999; 10: 129-136

74 Turmel-Rodrigues L, Sapoval M, Pengloan J et al. Manual thromboaspiration and dilation of thrombosed dialysis access: mid-term results of a simple concept. J Vasc Interv Radiol 1997; 8: 813-824

75 Liang H-L, Pan H-B, Chung H-M et al. Restoration of thrombosed Brescia-Cimino dialysis fistulas by using percutaneous transluminal angioplasty. Radiology 2002; 223: 339-344

76 Yang CC, Yang CW, Wen SC et al. Comparisons of clinical outcomes for thrombectomy devices with different mechanisms in hemodialysis arteriovenous fistulas. Catheter Cardiovasc Interv 2012; 80: 1035-1041

77 Dougherty MJ, Calligaro KD, Schindler $N$ et al. Endovascular versus surgical treatment for thrombosed hemodialysis grafts: A prospective, randomized study. J Vasc Surg 1999; 30: 1016-1023

78 Safa AA, Valji K, Roberts AC et al. Detection and treatment of dysfunctional hemodialysis access grafts: effect of a surveillance program on graft patency and the incidence of thrombosis. Radiology 1996; 199: $653-657$

79 Karnabatidis D, Kitrou P, Spiliopoulos S et al. Stent-grafts versus angioplasty and/or bare metal stents for failing arteriovenous grafts: a crossover longitudinal study. J Nephrol 2013; 26: 389-395

80 Haskal ZJ, Trerotola S, Dolmatch B et al. Stent graft versus balloon angioplasty for failing dialysis-access grafts. N Engl J Med 2010; 362: 494-503

81 Prologo JD, Minwell G, Kent J et al. Effect of the time to intervention on the outcome of thrombosed dialysis access grafts managed percutaneously. Diagn Interv Radiol 2014; 20: 143 - 146

82 Kakisis JD, Avgerinos E, Giannakopoulos T et al. Balloon angioplasty vs nitinol stent placement in the treatment of venous anastomotic stenoses of hemodialysis grafts after surgical thrombectomy. J Vasc Surg 2012; 55: $472-478$

83 Aruny JE, Lewis CA, Cardella JF et al. Quality improvement guidelines for percutaneous management of the thrombosed or dysfunctional dialysis access. Standards of Practice Committee of the Society of Cardiovascular \& Interventional Radiology. J Vasc Interv Radiol 1999; 10 $491-498$

84 Trerotola SO, Vesely TM, Lund GB et al. Treatment of thrombosed hemodialysis access grafts: Arrow-Trerotola percutaneous thrombolytic device versus pulse-spray thrombolysis. Arrow-Trerotola Percutaneous Thrombolytic Device Clinical Trial. Radiology 1998; 206: 403-414

85 Smits HFM, Smits JHM, Wüst AFJ et al. Percutaneous thrombolysis of thrombosed haemodialysis access grafts: comparison of three mechanical devices. Nephrol Dial Transplant Off Publ Eur Dial Transpl Assoc - Eur Ren Assoc 2002; 17: 467-473

86 Mathias $K$, Jäger $H$, Willaschek J et al. Interventional radiology in central venous obstructions. Dilatation - stent implantation- thrombolysis. Radiol 1998; 38: 606-613

87 Shi $Y$, Cheng J, Song $Y$ et al. Anatomical factors associated with left innominate vein stenosis in hemodialysis patients. Hemodial Int Int Symp Home Hemodial 2014; DOI: 10.1111/hdi.12131

88 Jaberi A, Schwartz D, Marticorena $R$ et al. Risk factors for the development of cephalic arch stenosis. J Vasc Access 2007; 8: 287-295

89 Ozyer $U$, Harman A, Yildirim $E$ et al. Long-term results of angioplasty and stent placement for treatment of central venous obstruction in 126 hemodialysis patients: a 10-year single-center experience. Am J Roentgenol 2009; 193: 1672 - 1679

90 Shemesh D, Goldin I, Zaghal I et al. Angioplasty with stent graft versus bare stent for recurrent cephalic arch stenosis in autogenous arteriovenous access for hemodialysis: a prospective randomized clinical trial. J Vasc Surg 2008; 48: 1524-1531, 1531.e1-2

91 Turmel-Rodrigues LAE. Regarding "Angioplasty with stent graft versus bare stent for recurrent cephalic arch stenosis in autogenous arteriovenous access for hemodialysis: A prospective randomized clinical trial". J Vasc Surg 2009; 50: 472

92 Bhatia DS, Money SR, Ochsner JL et al. Comparison of surgical bypass and percutaneous balloon dilatation with primary stent placement in the treatment of central venous obstruction in the dialysis patient: one-year follow-up. Ann Vasc Surg 1996; 10: 452-455

93 Mickley V, Görich J, Rilinger $N$ et al. Stenting of central venous stenoses in hemodialysis patients: long-term results. Kidney Int 1997; 51: 277 280 